\title{
Uncertainties in the Solar Neutrino Flux
}

\author{
W.C. Haxton ${ }^{\mathrm{a}}$

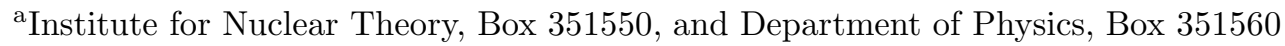 \\ University of Washington, Seattle, WA 98195, USA
}

\begin{abstract}
I discuss three issues relevant to solar neutrino flux measurements: cross section uncertainties in pp chain reactions, uncertainties in the GALLEX/SAGE response to ${ }^{7} \mathrm{Be}$ and ${ }^{51} \mathrm{Cr}$ neutrinos, and the implications of helioseismology for nonstandard suns with mixed cores. A few comments are also offered on $\nu_{e} \leftrightarrow \nu_{\tau}$ oscillations, cosmologically interesting neutrino masses, and recent proposals for supernova neutrino observatories.
\end{abstract}

\section{INTRODUCTION}

It is a pleasure to be present for this historic meeting hosted by the Superkamiokande collaboration. In this talk I will address three issues affecting the solar neutrino flux and one connected with future detectors for supernova neutrinos.

\subsection{Nuclear Physics of the pp Chain}

One of the crucial inputs into the solar model is the network of nuclear reactions comprising the pp chain (and CNO cycle). This network involves nonresonant charged particle reactions occurring at center-of-mass energies well below the height of the Coulomb barrier. As the solar core temperature $T_{c} \sim 1.5 \cdot 10^{7} \mathrm{~K}$, the typical kinetic energy for a nucleus in the core is $\langle E>\sim 2 \mathrm{keV}$. The competition between the Coulomb barrier and Boltzman distribution leads to a typical energy for reacting nuclei of $\left\langle E_{\text {reacting }}\right\rangle \sim 10 \mathrm{keV}$, a value that is generally lower than that where such reactions can be measured in the laboratory. Thus the task for nuclear physicists is to measure such reactions as accurately as possible over the accessible range of laboratory energies, then extrapolate these measurements to the energies relevant for the sun.

In the case of the driving reaction of the pp chain

$p+p \rightarrow{ }^{2} \mathrm{H}+\mathrm{e}^{+}+\nu_{\mathrm{e}}$,

the cross section is not measurable in the laboratory. Thus we must rely on theory. Fortunately deuterium is the simplest nucleus, and its properties are very well reproduced by NN poten- tials (Bonn, Paris, Argonne v18, etc.) carefully fit to phase shifts. The calculated cross section depends on the accuracy with which the axial vector coupling $g_{A}$ is known and on two-body corrections to the space-like component of the axial current, which are fortunately of order $(\mathrm{v} / \mathrm{c})^{2} \sim$ $1 \%$, where $\mathrm{v}$ is a typical bound nucleon velocity.

The other major reactions of the pp chain are measureable, but generally not at the low energies relevant to our sun. The necessary extrapolation of the cross section $\sigma(E)$ to lower energies is accomplished via the $\mathrm{S}$ factor

$\sigma(E) \equiv \frac{S(E)}{E} e^{-2 \pi Z_{1} Z_{2} \alpha / \beta}$

where $E$ is the center-of-mass energy, $Z_{1}$ and $Z_{2}$ are the charges of the interacting nuclei, and $\beta$ is the relative velocity. The introduction of $S(E)$ removes the s-wave Coulomb interaction of point particles and thus provides a much smoother quantity for use in extrapolating data. $S(E)$ depends on a number of physical effects - nuclear finite size, atomic screening corrections, higher particle waves, etc. - that the theorist must evaluate before this extrapolation can be done.

Because the solar neutrino problem is at a crucial juncture, a group of about 40 experts recently met at the Institute for Nuclear Theory, Seattle, to discuss the nuclear physics of the pp chain and $\mathrm{CNO}$ cycle. The questions addressed included the best current values for cross sections, critiques of assigned uncertainties, and recommendations for future experimental and theoretical work that could further improve our understanding of the 
nuclear physics. The summary of this workshop will appear in Reviews of Modern Physics (October, 1998) and is also available on the LANL preprint archive [1].

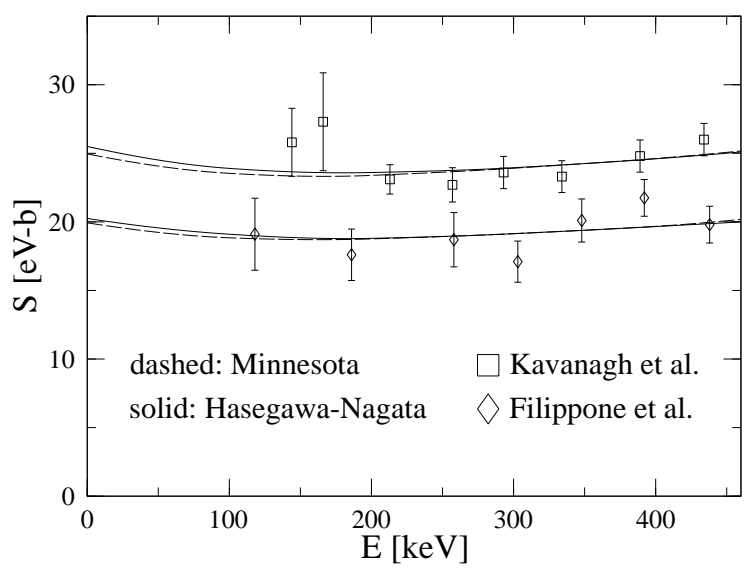

Figure 1. The ${ }^{7} \mathrm{Be}(p, \gamma){ }^{8} \mathrm{~B}$ S-factor as measured by Filippone et al. [3] and by Kavanagh et al. [4]. For each data set two theoretical extrapolations to $\mathrm{S}(0)$, reflecting different choices for the strong potential, are shown [2].

While I cannot give an adequate summary here, I will mention two of the reactions where significant changes were recommended. The first of these is ${ }^{7} \mathrm{Be}(\mathrm{p}, \gamma){ }^{8} \mathrm{~B}$, where the standard $\mathrm{S}_{17}(0) \sim$ $22.4 \mathrm{eVb}$ is that given by Johnson et al [2]. Measurements of $\mathrm{S}_{17}(\mathrm{E})$ are complicated by the need to use radioactive targets and thus to determine the areal density of the ${ }^{7} \mathrm{Be}$ target nuclei. Two techniques have been employed, measuring the rate of $478 \mathrm{keV}$ photons from ${ }^{7} \mathrm{Be}$ decay or counting the daughter ${ }^{7} \mathrm{Li}$ nuclei via the reaction ${ }^{7} \mathrm{Li}(\mathrm{d}, \mathrm{p})^{8} \mathrm{Li}$. The low-energy data sets $[3,4]$ for $\mathrm{S}_{17}(\mathrm{E})$ disagree by $25 \%$, a systematic effect apparent in Fig. 1. Each data set is consistent with theory in its dependence on $\mathrm{E}$ : this dependence is simple in the illustrated low energy region as it is determined by the asymptotic nuclear wave function.

The Seattle working group on $\mathrm{S}_{17}(\mathrm{E})$ found that only one low-energy data set, that of Fil- ippone et al. [3], was described in the published literature in sufficient detail to be evaluated. The target activity in that experiment had been measured by both $478 \mathrm{keV}$ gamma rays and by the $(\mathrm{d}, \mathrm{p})$ reaction, with consistent results. The resulting recommended value was thus based on this measurement, yielding

$S_{17}(0)=19_{-2}^{+4} e V b, 1 \sigma$.

Since the workshop, two developments have occurred. The Orsay/Bordeaux/Paris-Sud/ USTHB group published [5] a new measurement of $\mathrm{S}_{17}(0)=18.5 \pm 1.0 \mathrm{eV}$ b, while a preliminary value from the Weizmann/Troitzk/Mainz/Isolde group of $\mathrm{S}_{17}\left(E_{p}=1.2 \mathrm{MeV}\right)=22.5 \pm 2.5 \mathrm{eV} \mathrm{b}$ has been announced [6].

The ${ }^{3} \mathrm{He}(\alpha, \gamma)^{7} \mathrm{Be}$ reaction has been measured by two techniques, by counting the capture $\gamma$ rays and by detecting the resulting ${ }^{7}$ Be activity. While the two techniques have been used by several groups and have yielded separately consistent results, the capture $\gamma$ ray value $\mathrm{S}_{17}(0)=0.507 \pm$ $0.016 \mathrm{keV}$ b is not in good agreement with the ${ }^{7} \mathrm{Be}$ activity value $0.572 \pm 0.026 \mathrm{keV}$-b. The Seattle working group concluded that the evidence for a systematic discrepancy of unknown origin was reasonably strong and recommended that standard procedures be used in assigning a suitably expanded error. The recommended value $\mathrm{S}_{34}(0)$ is $0.53 \pm 0.05$.

These and other recommended values were recently incorporated into the Bahcall and Pinnsoneault (BP98) solar model calculation [7]. While the workshop's recommended values involve no qualitative changes, there is some broadening of error bars and a downward shift in $\mathrm{S}_{17}(0)$, leading to the lower BP98 ${ }^{8} \mathrm{~B}$ flux. The workshop's Reviews of Modern Physics article summarizes a substantial amount of work on topics not discussed here: screening effects, weak radiative corrections to and exchange current effects on $\mathrm{p}+\mathrm{p}$, the atomic physics of ${ }^{7} \mathrm{Be}+\mathrm{e}^{-}$, etc. Much of this discussion was useful in evaluating possible uncertainties in solar microphysics, and in identifying opportunities for reducing those uncertainities. 


\subsection{The Nuclear Physics of the GALLEX/SAGE ${ }^{51}$ Cr Calibrations}

The ${ }^{51} \mathrm{Cr}$ neutrino source experiments provide an important check on the overall gallium detector operations under few atom, hot chemistry conditions. The issue discussed here, and which was mentioned in the earlier experimental talks, is the potential complication due to contributions of uncertain strength to the $5 / 2^{-}$and $3 / 2^{-71} \mathrm{Ge}$ excited states (see Fig. 2).

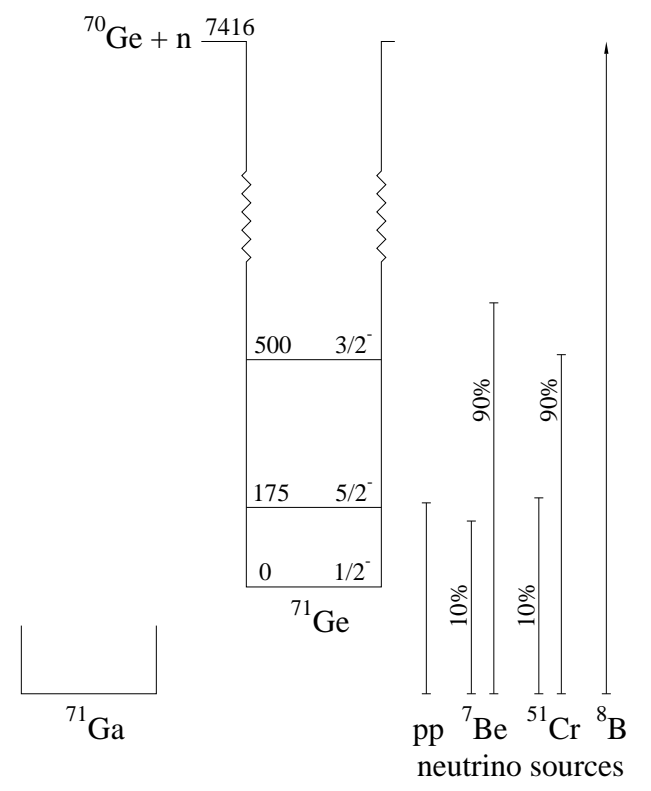

Figure 2. Level scheme for ${ }^{71} \mathrm{Ge}$ showing the excited states that contribute to absorption of $\mathrm{pp}$, ${ }^{7} \mathrm{Be},{ }^{51} \mathrm{Cr}$, and ${ }^{8} \mathrm{~B}$ neutrinos.

The results of the source experiments can be normalized to the known ${ }^{71} \mathrm{Ge}$ ground state contribution [8], yielding

$$
\begin{aligned}
& R_{o}=E\left[1+0.67 \frac{\mathrm{BGT}\left(5 / 2^{-}\right)}{\mathrm{BGT}(\mathrm{gs})}+0.22 \frac{\mathrm{BGT}\left(3 / 2^{-}\right)}{\mathrm{BGT}(\mathrm{gs})}\right] \\
& = \begin{cases}0.98 \pm 0.08, & \text { GALLEX }[9] \\
1.00 \pm 0.13, & \text { SAGE }[10]\end{cases}
\end{aligned}
$$

where $E$ represents any departure of the efficiency from the value determined from tracers and used by the experimentalists in their analyses. The dependence of the results on the unknown BGT values is explicit. Clearly the conclusion $E \sim 1$ requires an independent determination that the unknown BGT values are much smaller than the ground state value $\mathrm{BGT}(\mathrm{gs})$.

It had been assumed that forward-angle $(p, n)$ charge exchange measurements determine the unknown BGT values

$$
\begin{aligned}
& \frac{\operatorname{BGT}\left(5 / 2^{-}\right)}{\operatorname{BGT}(\mathrm{gs})} \lesssim 0.06 \\
& \frac{\mathrm{BGT}\left(3 / 2^{-}\right)}{\mathrm{BGT}(\mathrm{gs})}=0.12 \pm 0.02
\end{aligned}
$$

But extensive investigations [11,12] of the proportionality between $(\mathrm{p}, \mathrm{n})$ cross sections and known weak interaction BGT values have shown that the relationship is a complicated one. Existing discrepancies can be removed by the assumption that forward-angle $(\mathrm{p}, \mathrm{n})$ effective operator contains a spin-tensor contribution of relative strength $\delta \sim 0.1$, in addition to the Gamow-Teller operator,

$\hat{O}_{(p, n)}=\sigma(i) \tau_{+}(i)+$

$\delta \sqrt{8 \pi}\left[Y_{2}\left(\Omega_{i}\right) \otimes \sigma(i)\right]_{J=1} \tau_{+}(i)$.

Simple considerations of the nuclear structure of ${ }^{71} \mathrm{Ga}$ and ${ }^{71} \mathrm{Ge}$ suggest that the tensor operator might be particularly troublesome for the ${ }^{71} \mathrm{Ga}\left(3 / 2^{-}\right) \rightarrow{ }^{71} \mathrm{Ge}\left(5 / 2^{-}\right)$transition to the first excited state. The naive description of this transition is

$1 f_{5 / 2}(n) \rightarrow 2 p_{3 / 2}(p)$,

an $\ell$-forbidden amplitude that generates an enormous spin-tensor and vanishing Gamow-Teller contributions. Well-known transitions of a similar character, e.g. to the first excited state in ${ }^{39} \mathrm{~K}(\mathrm{p}, \mathrm{n}){ }^{39} \mathrm{Ca}$, have produced discrepancies between $(\mathrm{p}, \mathrm{n})$ and weak interaction transition probabilities of factors of $\sim 100$. The conclusion [8] is that it might be unwise to use the ${ }^{71} \mathrm{Ga}(\mathrm{p}, \mathrm{n})$ results as a reliable independent measurement of $\operatorname{BGT}\left(5 / 2^{-}\right)$.

To explore this further, I did a large-basis shell model calculation [8] of the ${ }^{71} \mathrm{Ga} \rightarrow{ }^{71} \mathrm{Ge}$ weak 
and $(\mathrm{p}, \mathrm{n})$ transitions. The results agree reasonably with what is known experimentally: the calculated BGT (g.s.) $=0.051$, compared to the experimental value 0.087 , while the calculated $(\mathrm{p}, \mathrm{n})$ BGT $\left(5 / 2^{-}\right)$, corresponding to the operator in Eq. (6), is 0.0006 , in agreement with the experimental bound $<0.005$. However the latter result stemmed from a cancellation between the GT and spin-tensor operators comprising $\mathrm{O}_{(\mathrm{p}, \mathrm{n})}$,

$$
\left\langle 5 / 2^{-}\left\|O_{p, n}\right\| 3 / 2^{-}\right\rangle=0.264-\delta 2.23 .
$$

The cancellation between the second term an enormous spin-tensor amplitude - and the GT amplitude leads to a much larger beta decay $\operatorname{BGT}\left(5 / 2^{-}\right)$than would be allowed by in a more naive interpretation of the (p,n) BGT value. Thus this is an explicit demonstration that previous bounds on BGT $\left(5 / 2^{-}\right)$are too aggressive.

It is argued in Ref. [8] that, by relying on the shell model calculation of the very strong tensor amplitude in Eq. (8), a reasonable range of beta decay BGT $\left(5 / 2^{-}\right)$can be extracted from the $(\mathrm{p}, \mathrm{n})$ measurements. This results in a corresponding change in the ${ }^{51} \mathrm{Cr}$ cross section from the previous standard value

$\sigma\left({ }^{51} \mathrm{Cr}\right)=\left(5.81_{-0.16}^{+0.21}\right) \cdot 10^{-45} \mathrm{~cm}^{2}$

to

$\sigma\left({ }^{51} \mathrm{Cr}\right)=(6.39 \pm 0.68) \cdot 10^{-45} \mathrm{~cm}^{2}$

where the error in Eq. (9b) represents that due to excited state uncertainties only. If this value is used in Eq. (4), one finds

$E= \begin{cases}0.86 \pm 0.07 \pm 0.09, & \text { GALLEX } \\ 0.875 \pm 0.11 \pm 0.09, & \text { SAGE }\end{cases}$

where the first uncertainty in the source experiment error while the second corresponds to the ${ }^{51} \mathrm{Cr}$ cross section. Note that $\mathrm{E} \sim 1$ is allowed, though it is certainly not demanded. It is important to note that the difference between (9a) and $(9 \mathrm{~b})$ is one of an extended error range: all of the range in $(9 \mathrm{a})$ that is attributable to excited state uncertainties is allowed in (9b). It is also notable that the cross section uncertainty in Eq. (10) is comparable to the source experiment uncertainty.

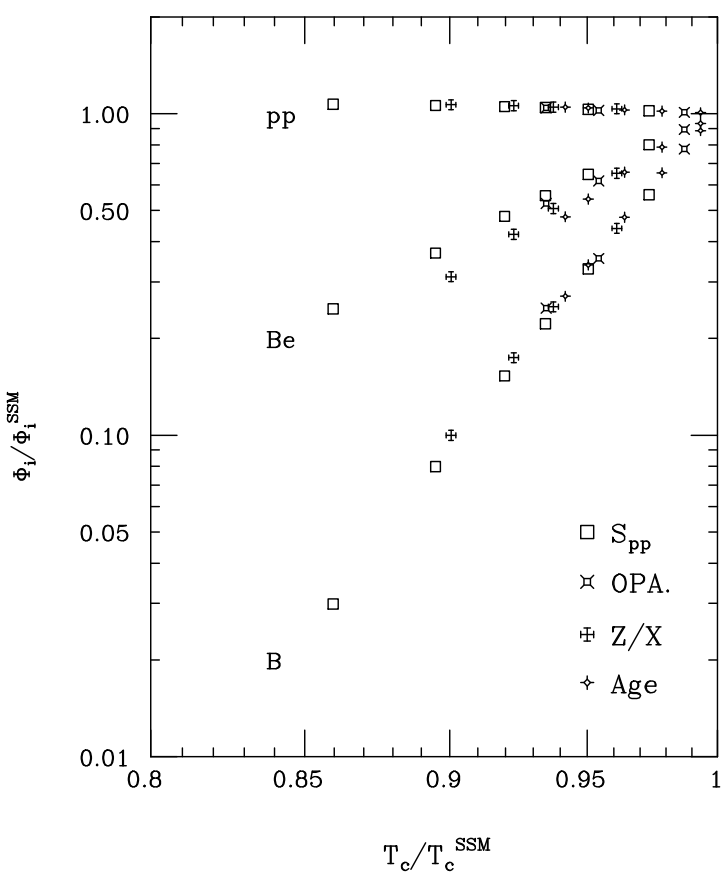

Figure 3. The response of the $\mathrm{pp}, \mathrm{Be}$, and $\mathrm{B}$ neutrino fluxes to the indicated variations in solar model input parameters, displayed as a function of the resulting central temperature $\mathrm{T}_{c}$. (From Castellani et al. [13].)

The conclusion is that the source experiments have become ${ }^{7} \mathrm{Be}$ neutrino cross section measurements. Indeed, one can express the GALLEX/SAGE responses to the ${ }^{7} \mathrm{Be}$ neutrinos in terms of $R_{o}$, independent of almost all nuclear physics uncertainties. This means of course that other tests of $\mathrm{E} \sim 1$ under few-atom, hot chemistry conditions take on added importance. Thus the GALLEX ${ }^{71}$ As test discussed by Prof. Kirsten at this meeting is crucial. The GALLEX/SAGE results are central to the conclusions of global analyses of the solar neutrino experiments that yield $\phi\left({ }^{7} \mathrm{Be}\right) \lesssim 0$. 


\subsection{Solar Core Mixing of ${ }^{3} \mathrm{He}$ and Helio- seismology}

The crux of the solar neutrino problem can be captured in two experimental quantities. First the ${ }^{8} \mathrm{~B}$ neutrino flux $\phi\left({ }^{8} \mathrm{~B}\right)$, which varies approximately as $T_{c}^{18}$ where $T_{c}$ is the solar core temperature, is known to be reduced by about a factor of $1 / 3$ (0.47 using the new BP98 results). Naively this result requires a cooler sun,

$T_{c} \sim 0.96 T_{c}^{\mathrm{SSM}}$,

where the superscript SSM denotes the standard solar model result. However the flux ratio $\phi\left({ }^{7} \mathrm{Be}\right) / \phi\left({ }^{8} \mathrm{~B}\right)$, which varies as $T_{c}^{-10}$, also appears to be reduced relative to the standard model. This then requires

$T_{c}>T_{c}^{\mathrm{SSM}}$

with the extent of the increase depending on how strongly one wants to suppress this ratio. $\left(\phi\left({ }^{7} \mathrm{Be}\right) \sim 0\right.$ provides the best fit to the ${ }^{37} \mathrm{Cl}$, GALLEX/SAGE, and Superkamiokande results.) It appears that the experimental results on $\phi\left({ }^{8} \mathrm{~B}\right)$ and $\phi\left({ }^{7} \mathrm{Be}\right) / \phi\left({ }^{8} \mathrm{~B}\right)$ are thus in conflict, with the first requiring a cooler sun and the second a hotter one.

These arguments depend on the assumption that neutrino fluxes will track $T_{c}$ as described above, but this appears to hold remarkably well. Figure 3, from Castellani et al. [13], illustrates this: changes due to modified nuclear cross sections, opacity, lowered metallicity, and the solar age produce neutrino fluxes that track the result$\operatorname{ing} T_{c}$ quite accurately. This led some in the field to argue that no nonstandard solar model could produce the observed pattern of neutrino fluxes.

Andrew Cumming and I decided to test this claim phenomenologically, under the assumption of a steady-state sun with conventional microphysics producing the correct luminosity. As our procedures are described elsewhere [14], I'll just state here our basic result. There appears to be only one possibility for constructing a steady state model with a neutrino flux pattern reasonably close to experiment: the solar core must mix on timescales of ${ }^{3} \mathrm{He}$ equilibration $(\sim$ few $10^{6}$ years) in the "elevator convection" pattern illustrated in Fig. 4. This mixing produces

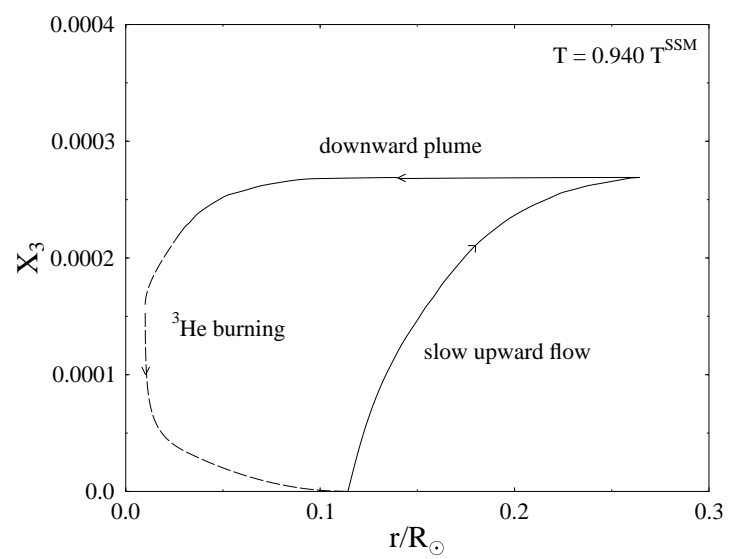

Figure 4. A phenomenologically derived core convection pattern that will suppress both $\phi\left({ }^{8} \mathrm{~B}\right)$ and $\phi\left({ }^{7} \mathrm{Be}\right) / \phi\left({ }^{8} \mathrm{~B}\right)[14]$. The downward flow is in plumes, rapid and localized, requiring $\sim$ few $\cdot 10^{6}$ years. This leads to out-of-equilibrium burning of ${ }^{3} \mathrm{He}$ at small $\mathrm{r}$. The slow, broad, upward flow allows the cycle to replenish the ${ }^{3} \mathrm{He}$. Typical upward times are $\sim$ few $\cdot 10^{7}$ years.

the desired flux pattern because it modifies the $\mathrm{ppI} /(\mathrm{ppII}+\mathrm{ppIII})$ and $\mathrm{ppII} / \mathrm{ppIII}$ branching ratios in the proper way. The former is enhanced because the resulting ${ }^{3} \mathrm{He}$ enrichment of the core favors the ${ }^{3} \mathrm{He}+{ }^{3} \mathrm{He}$ reaction. The latter is reduced because the fraction of ${ }^{3} \mathrm{He}$ that burns by ${ }^{3} \mathrm{He}+{ }^{4} \mathrm{He}$ produces ${ }^{7} \mathrm{Be}$ deep in the core, where the higher temperatures favor ppIII over ppII.

This exercise indicated that arguments against nonstandard solar model solutions based on how neutrino fluxes scale with $T_{c}$ are not completely general. While the pattern of core mixing was derived phenomenologically, and not on physical grounds, it nevertheless has some physical appeal. The possibility of core mixing generated by the standard solar model overstability in the ${ }^{3} \mathrm{He}$ gradient was first discussed by Dilke and Gough. Roxburgh discussed a persistent convective core as a possible consequence of the growth of the SSM ${ }^{3} \mathrm{He}$ gradient during our sun's early convective stage.

Several astrophysical consequences of such mix- 
ing were discussed, with one, helioseismology, appearing problematic. Bahcall et al. [15] and Fiorentini et al. [16] evaluated the helioseismological consequences of replacing the SSM core molecular weight profile by a constant one, such as would occur for a continuously mixed model. This yielded $8 \%$ deviations in core sound speeds, far outside allowable bounds. My summary of this work is that it might be viewed as an attempt to estimate the natural scale of expected helioseismology changes. However, it does not convincingly settle the issue because such a modification of the SSM produces a "model" that fails to satisfy the equations of stellar evolution. One can envision that it might be more difficult to change the sound speed profile $\mathrm{c}(\mathrm{r})$ in a dynamically consistent model where the pressure and density profiles are coupled through the condition of hydrostatic equilibrium.

A stronger argument, which arose from discussions at last December's ITP conference on solar neutrinos, is that existing helioseismology determinations of $\mathrm{c}(\mathrm{r})$ coupled with a constant molecular weight profile in the core would necessarily lead to an unphysical temperature profile $\mathrm{T}(\mathrm{r})$ that would increase away from $\mathrm{r}=0$. It appears this conclusion is overstated: adiabatically mixed models lacking molecular weight gradients in the core generically have profiles in $\mathrm{T}(\mathrm{r})$ that are convex downward. [While this counterargument to the ITP discussions was originally made to me by Richard Epstein, similar remarks have been made by John Bahcall and by Doug Gough at this conference.]

My view is that such mixed models, while quite unlikely, are not yet definitively ruled out. The issue of their viability is an important one, given the argument that a core mixed on the ${ }^{3} \mathrm{He}$ equilibration timescale is the only possibility for producing acceptable neutrino fluxes in a steady state model. Plans are underway at Los Alamos to evolve a series of $1 \mathrm{D}$ models where mixing is included through mixing length theory, adjusting these in the usual way to produce the proper luminosity after 4.6 b.y. of the stellar burning. Helioseismology studies performed on these models should then settle the issue.

\subsection{Supernova $\nu_{e} \leftrightarrow \nu_{\tau}$ Oscillations and Cosmologically Interesting Neutrino Masses}

In a Type II supernova $99 \%$ of the energy released by the core collapse is carried off by neutrinos. The initial flavor equilibrium of neutrinos trapped within the core at densities $\rho \gtrsim 10^{12}$ $\mathrm{g} / \mathrm{cm}^{3}$, coupled with the flavor-dependent decoupling of neutrinos from the matter at the neutrinosphere, leads to an approximate equipartition of energy among the flavors and to a characteristic hierarchy of temperatures. The average energy of heavy flavor neutrinos $\left\langle E_{\nu_{\mathrm{HEAVY}}}\right\rangle \sim$ $25 \mathrm{MeV}$, while $\left\langle E_{\bar{\nu}_{e}}\right\rangle \sim 16 \mathrm{Mev}$ and $\left\langle E_{\nu_{e}}\right\rangle \sim$ $11 \mathrm{MeV}$. The lower values for the $\nu_{e} \mathrm{~s}$ and $\bar{\nu}_{e} \mathrm{~s}$ reflects their stronger matter couplings due to charged current reactions with nucleons and to their greater scattering cross sections off electrons. The lower $\nu_{e}$ temperature, relative to $\bar{\nu}_{e}$, is due to the neutron richness of the matter near the neutrinosphere and resulting enhancement of $\nu_{e}+n \rightarrow p+e^{-}$. The neutrino energy hierarchy $\left\langle E_{\nu_{\mathrm{HEAVY}}}\right\rangle>\left\langle E_{\bar{\nu}_{e}}\right\rangle>\left\langle E_{\nu_{e}}\right\rangle$ appears to be a result independent of the details of supernova modelling, in contrast to the case of solar neutrinos where fluxes depend on nuclear reaction networks.

The spectrum of neutrinos is essentially fixed at the neutrinosphere, $\rho \sim 10^{12} \mathrm{~g} / \mathrm{cm}^{3}$, a density that corresponds to a neutrino oscillation level crossing for $\delta m^{2} \sim 10^{4} \mathrm{eV}^{2}$. Furthermore, the density scale height at this density will produce an adiabatic neutrino level crossing for $\sin ^{2} 2 \theta \gtrsim$ $10^{-5}$. Thus the supernova neutrino spectrum provides a unique opportunity to probe neutrino oscillations. In particular, if the neutrino masses have a seesaw pattern

$m_{\nu} \propto \frac{m_{D}^{2}}{M_{R}} \quad m_{D}^{2} \leftrightarrow m_{u}^{2}: m_{c}^{2}: m_{t}^{2}$

one can fix $M_{R}$ according to the solar neutrino small angle solution, assuming the solar neutrino puzzle is due to $\nu_{e} \rightarrow \nu_{\mu}$. Thus " $m_{\nu_{\mu}}$ " $\sim$ few $\cdot 10^{-3} \mathrm{eV}$ and " $m_{\nu_{\tau}}$ " $\sim 1 \mathrm{eV}$, a value that would be interesting cosmologically and would induce $\nu_{e} \leftrightarrow \nu_{\tau}$ oscillations for supernova neutrinos. This is illustrated in Fig. 5. As a consequence of this crossing, supernova $\nu_{e}$ s will emerge anoma- 


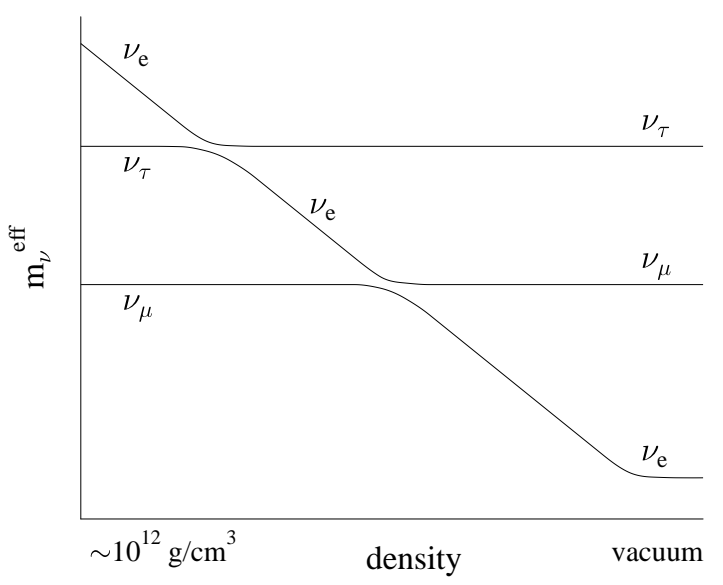

Figure 5. The three-flavor level crossing diagram showing two "crossings" that might be associated with matter enhanced oscillations of supernova neutrinos.

lously hot, with an $\left\langle E_{\nu_{e}}\right\rangle \sim 25 \mathrm{MeV}$ characteristic of heavy flavor neutrinos. An experimental demonstration that $\left\langle E_{\nu_{e}}\right\rangle>\left\langle E_{\bar{\nu}_{e}}\right\rangle$, for example, would provide strong evidence for oscillations and possibly provide information on massive tauon neutrinos.

Such an oscillation would have consequences for the supernova explosion, as it would enhance matter heating by the $\nu_{e} \mathrm{~s}$, and on nucleosynthesis, as the increased rate for $\nu_{e}+n \rightarrow e^{-}+p$ would drive the atmosphere above the protoneutron star proton rich, destroying any possibility for an $\mathrm{r}$ process. But the aspect on which I would like to focus is the possibility of distinctive oscillation signals in terrestrial supernova neutrino observatories.

One detector of interest, despite being primarily sensitive to $\bar{\nu}_{e} \mathrm{~s}$, is Superkamokande. The usual $\nu_{e}$ signal, elastic scattering off electrons, will not be altered in total rate due to a $\nu_{e} \leftrightarrow$ $\nu_{\tau}$ oscillation, since this rate is proportional to the luminosity, which is approximately independent of flavor. However there is some hardening of the spectrum of forward-scattered electrons: the question is whether this is enough of a sig- nal, given uncertainties in the supernova $\nu_{e}$ and $\nu_{\mu} / \bar{\nu}_{\mu} / \nu_{\tau} / \bar{\nu}_{\tau}$ fluxes contributing to this forward scattering of electrons [17]. Perhaps more interesting is the reaction $\nu_{e}+{ }^{16} \mathrm{O} \rightarrow{ }^{16} \mathrm{~F}+e^{-}$, which produces a backward-peaked distribution of electrons that would very likely be detectable above the $\overline{\nu_{e}}$ "background," given $\left\langle E_{\nu_{e}}\right\rangle \sim 25 \mathrm{MeV}[18]$. There has also been a suggestion that the $\gamma$-ray cascades following $\nu$-induced spallation reactions on ${ }^{16} \mathrm{O}$ might provide an attractive signal [19].

It seems to me that a detector of a different type - one flavor specific and economical to operate - might be useful in supernova watches, given that the characteristic time between galactic events might be $\sim 35$ years. An attractive possibility is the 1 kiloton version of the iodine detector discussed by Lande, which would be able to view the entire galaxy with good statistics. The cross section averaged over the $\nu_{e}$ flux is predicted to increase by a factor of $\sim 6.6$ if there is a $\nu_{e} \leftrightarrow \nu_{\tau}$ oscillation. As the luminosity constraint leads to a smaller flux of (undistorted) $\nu_{\tau} \mathrm{s}$, this implies an increase in the iodine detector response of a factor of $\sim 2.9$, a rather dramatic signal for oscillations.

Another possibility is the lead neutron spallation detector LAND proposed by Hargrove et al. [20]. The signal he discussed, single neutron emission, is not flavor specific nor is the cross section well determined. However, Fuller, McLaughlin, and I [21] recently found a rather attractive charge-current-specific signal in this detector, the emission of multiple neutrons. Due to details of the nuclear physics - the location of the giant resonances that are strongly excited by charged and neutral current scattering - this channel appears to "filter out" neutral current events, while leaving $\sim 70 \%$ of the charged current response. Thus if multiple neutron events are studied, LAND becomes flavor specific and, due to the high threshold for reaching the giant resonances, extraordinarily sensitive to the $\nu_{e}$ temperature. A $\nu_{e} \leftrightarrow \nu_{\tau}$ oscillation would produce approximately 40 times the number of events that would be measured in the absence of oscillations. The cross section for detecting $\left\langle E_{\nu_{e}}\right\rangle \sim 25 \mathrm{MeV}$ supernova neutrinos is a remarkable $4 \cdot 10^{39} \mathrm{~cm}^{2}$. There is some work remaining to be done to verify these results, but the prospects appear quite promising. 
This conference is a wonderful illustration of the power of experiments with astrophysical neutrinos. Supernova neutrinos differ from atmospheric and solar neutrinos in that they impact the earth only once or twice a century. But they provide unique windows on neutrino physics, such as $\nu_{\tau}$ masses of cosmological interest. Thus it is probably important for us to prepare the right complement of neutrino observatories in anticipation of the next galactic supernova.

This work was supported in part by the U.S. Department of Energy.

\section{REFERENCES}

1. E.G. Adelberger, et al., to appear in Rev. Mod. Phys.

2. C.W. Johnson, E. Kolbe, S.E. Koonin, and K. Langanke, Ap. J, 392 (1992) 320.

3. B.W. Filippone, A. J. Elwyn, C. N. Davids, and D. D. Koetke, Phys. Rev. Lett., 50 (1983) 412 and Phys. Rev. C, 28 (1983) 2222.

4. R. W. Kavanagh, T. A. Tombrello, J. M. Mosher, and D. R. Goosman, Bull. Am. Phys. Soc., 14 (1969) 1209.

5. F. Hammache et al., Phys. Rev. Lett. 80 (1998) 928.

6. L. Weissman et al., Nucl. Phys. A, 630 (1998) 678; M. Hass et al., private communication.

7. J. N. Bahcall and M. H. Pinsonneault, Rev. Mod. Phys., 67 (1995) 781 and to be published (1998).

8. W. C. Haxton, Phys. Lett. B, 431 (1998) 110.

9. P. Anselmann, et al., Phys. Lett. B, 342 (1995) 440; W. Hampel et al., Phys. Lett. B, 388 (1996) 384.

10. D. N. Abdurashitov et al., Phys. Lett. B, 328 (1994) 234 and hep-ph/9803418 (March, 1998).

11. S.M. Austin, N. Anantaraman, and W. G. Love, Phys. Rev. Lett., 73 (1994) 30; J.W. Watson et al., Phys. Rev. Lett., 55 (1985) 1369.

12. N. Hata and W. C. Haxton, Phys. Lett. B, $353,(1995) 422$.

13. V. Castellani, S. Degl'Innocenti, G. Fiorentini, M. Lissia, and B. Ricci, Phys. Rev. D, 50 (1994) 4749.
14. A. Cumming and W. C. Haxton, Phys. Rev. Lett., 77 (1996) 4286.

15. J.N. Bahcall, M. H. Pinsonneault, S. Basu, and J. Christensen-Dalsgaard, Phys. Rev. Lett., 78 (1997) 171.

16. G. Fiorentini, talk presented on the ITP Workshop on Solar Neutrinos, December 1997 http://www.itp.ucsb.edu/online/snu fiorentini).

17. H. Minakata, private communication.

18. W.C. Haxton, Phys. Rev. D, 36 (1987) 2283; Y. Z. Qian and G.M. Fuller, Phys. Rev. D, 49 (1996) 1762.

19. K. Langanke, P. Vogel, and E. Kolbe, Phys Rev. Lett., 76 (1997) 2629.

20. C.K. Hargrove et al., Astroparticle Physics, 5 (1996) 183.

21. G.M. Fuller, W. C. Haxton and G. C. McLaughlin, submitted to Phys. Rev. D. 